\title{
Modeling nano-scale grain growth of intermetallics
}

\author{
MOHSEN KAZEMINEZHAD \\ Department of Materials Science and Engineering, Sharif University of Technology, Azadi Avenue, Tehran, Iran
}

MS received 22 June 2008; revised 13 August 2008

\begin{abstract}
The Monte Carlo simulation is utilized to model the nano-scale grain growth of two nanocrystalline materials, $\mathrm{Pd}_{81} \mathrm{Zr}_{19}$ and $\mathrm{RuAl}$. In this regard, the relationship between the real time and the time unit of simulation, i.e. Monte Carlo step (MCS), is determined. The results of modeling show that with increasing time of heating, the grain sizes of both nano-crystalline materials increased as in the case of conventional materials. Moreover, it is found that for both nano-crystalline materials the relationship between the real time and MCS is in power law form, which is linear for the conventional materials.
\end{abstract}

Keywords. Nanostructured intermetallics; grain growth; simulation; Monte Carlo.

\section{Introduction}

The grain growth phenomenon is a topic of much interest in materials science. The grain size of materials can be changed by grain growth phenomenon which affects their mechanical properties. Grain growth occurs in polycrystalline materials to decrease the interfacial free energy of the system by reducing the total grain boundary energy (Christian 1965). The kinetics of grain growth can be expressed by the following empirical relationships (Christian 1965; Humphreys and Hatherly 1995)

$$
D=k_{1} t^{n}
$$

or

$$
D^{1 / n}-D_{0}^{1 / n}=k_{2} t,
$$

where $D$ is the grain size at time $t, D_{0}$ the initial grain size at $t=0, k_{1}, k_{2}$ and $n$ are the constants. For most of the conventional (coarse-grained) polycrystalline materials, the value of $n$ is within a range of $0.25-0.5$ and its typical value is $0 \cdot 5$. The nano-crystalline materials are structurally characterized by ultra fine grains. These grains are separated by grain boundaries. Thus, the volume fraction of the boundaries in nano-crystalline materials is higher than that in conventional polycrystalline materials (Liu and Kirchheim 2004). For nano-crystalline materials, the large change in total grain boundary area accompanying grain growth would greatly affect the grain growth kinetics. As grain boundary area diminishes, the concentration of solute atoms segregated to the grain boundaries is increased. Consequently, this introduces a grain size

(mkazemi@sharif.edu) dependent retarding force on grain boundary migration (Liu and Mucklich 2001; Liu et al 2006). Therefore, the kinetics of grain growth in nano-crystalline materials can rarely be described by the parabolic manner of that in conventional polycrystalline materials (Michels et al 1999).

However, Monte Carlo simulation has been widely used for modeling the grain growth of the conventional polycrystalline materials, it has not yet been utilized for that of the nano-crystalline ones (Anderson et al 1984; Morhac and Morhacova 2000). In the simulation, considering a linear relationship between the real time and time unit of simulation, i.e. Monte Carlo step (MCS), the real grain growth kinetics are taken for conventional polycrystalline materials (Saito and Enomoto 1992; Gao and Thompson 1996). Due to different grain growth kinetics of the conventional and nano-crystalline materials, it is expected that by modifying the conversion function of real time to MCS, the real grain growth kinetics can be taken in Monte Carlo simulation of the nano-scale polycrystalline materials. Therefore, this study is on the modeling of the nano-scale grain growth and finding the conversion function.

\section{Modeling the grain growth}

Monte Carlo simulation is utilized to model the grain growth phenomenon in nano-scale polycrystalline materials, $\mathrm{Pd}_{81} \mathrm{Zr}_{19}$ and $\mathrm{RuAl}$. A triangular lattice of $200 \times 200$ is generated and the random orientation numbers $S_{\mathrm{r}}$, $1 \leq S_{\mathrm{r}} \leq 64$, are assigned to each lattice point (Anderson et al 1984; Morhac and Morhacova 2000). Then a new random orientation number is assigned to a randomly selected point. The probability of reorientation at the lattice point is determined by evaluating the change of inter- 
facial energy, $\Delta E$, during reorientation (Gao and Thompson 1996):

$$
\begin{aligned}
& P=\left\{\begin{array}{ll}
0 & (\Delta E>0) \\
1 & (\Delta E \leq 0)
\end{array},\right. \\
& \Delta E=\sigma_{\mathrm{b}} \sum_{i=1}^{6}\left(\delta_{s_{i} s_{o}}-\delta_{s_{i} s_{n}}\right),
\end{aligned}
$$

where $P$ is the probability, $\sigma_{\mathrm{b}}$ the grain boundary energy, $S_{\mathrm{o}}$ the original orientation number at a lattice point, $S_{i}$ the orientation number of its six nearest neighbours, $S_{\mathrm{n}}$ a new random orientation number and $\delta$ the Kronecker function.

It should be mentioned that when the above procedure is repeated 40,000 times, a time unit of simulation, i.e. Monte Carlo step (MCS), is passed (Anderson et al 1984; Morhac and Morhacova 2000).

Generally, in single phase nano-crystalline materials the solute drag reduces the grain boundary mobility. On the other hand, the grain boundary energy, $\sigma_{\mathrm{b}}$, reduces with solute segregation (Liu and Kirchheim 2004; Liu et al 2006):

$$
\sigma_{\mathrm{b}}=\sigma_{0}-\Gamma_{\mathrm{b} 0}\left[R T \ln X_{0}+\Delta G_{\mathrm{seg}}\right]
$$

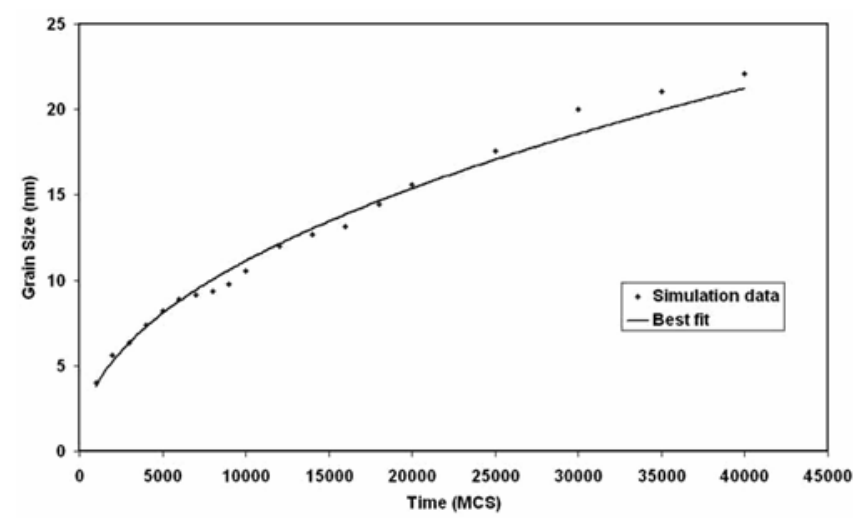

Figure 1. The simulated grain size of $\mathrm{Pd}_{81} \mathrm{Zr}_{19}$ at $873^{\circ} \mathrm{K}$ vs Monte Carlo step.

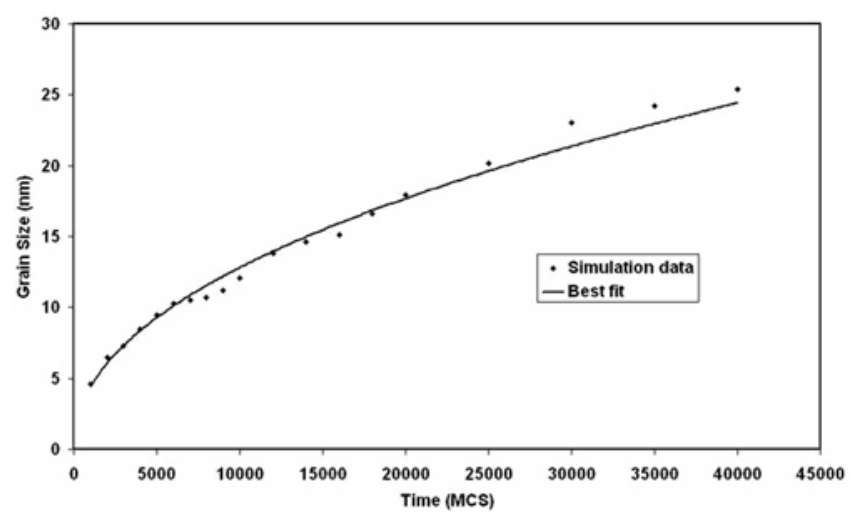

Figure 2. The simulated grain size of $\mathrm{RuAl}$ at $1173^{\circ} \mathrm{K}$ vs Monte Carlo step. where $\sigma_{0}$ and $\Gamma_{\mathrm{b} 0}$ are the constants, $R$ the gas constant, $T$ the temperature, $X_{0}$ the bulk concentration of solute and $\Delta G_{\text {seg }}$ the Gibbs free energy of segregation per mole of solute.

In a specific temperature the grain boundary energy can be expressed as follows (Liu and Kirchheim 2004):

$$
\sigma_{\mathrm{b}}=\sigma_{\mathrm{t}}-\Gamma_{\mathrm{b}} \Delta H_{\mathrm{seg}},
$$

where $\sigma_{\mathrm{t}}$ and $\Gamma_{\mathrm{b}}$ are the constants and $\Delta H_{\text {seg }}$ the segregation enthalpy.

Therefore, from (4) and (6) the energy change in Monte Carlo simulation of the nano-scale polycrystalline materials is given as follows:

$$
\Delta E=\left(\sigma_{\mathrm{t}}-\Gamma_{\mathrm{b}} \Delta H_{\mathrm{seg}}\right) \sum_{i=1}^{6}\left(\delta_{s_{i} s_{\mathrm{o}}}-\delta_{s_{i} s_{\mathrm{n}}}\right) .
$$

\section{Modeling results and discussion}

Utilizing the Monte Carlo simulation, the grain growth of two nano-crystalline materials, $\mathrm{Pd}_{81} \mathrm{Zr}_{19}$ at $873^{\circ} \mathrm{K}$ and $\mathrm{RuAl}$

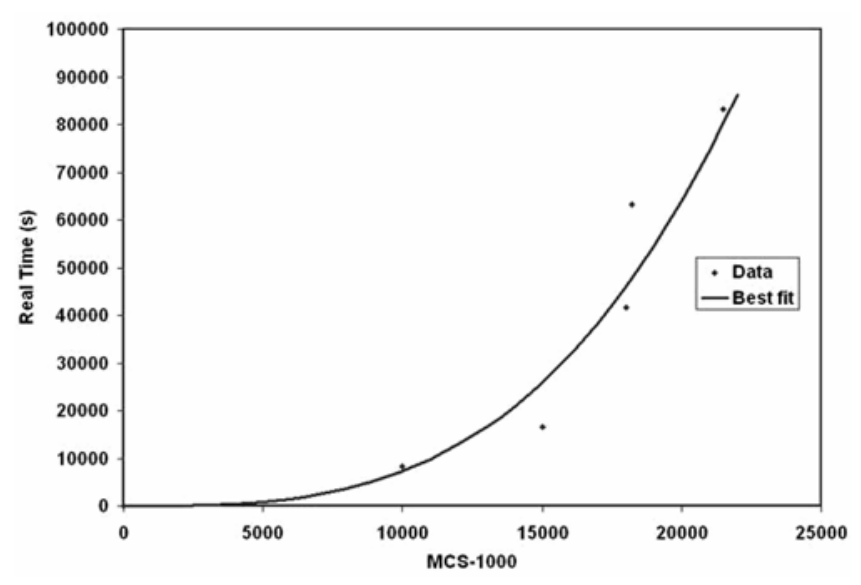

Figure 3. The real time vs Monte Carlo step for $\mathrm{Pd}_{81} \mathrm{Zr}_{19}$ at $873^{\circ} \mathrm{K}$.

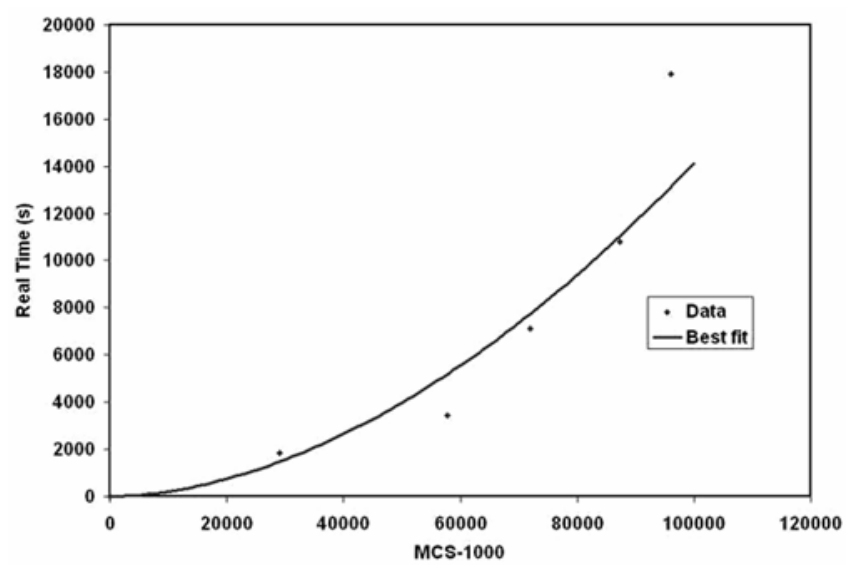

Figure 4. The real time vs Monte Carlo step for $\mathrm{RuAl}$ at $1173^{\circ} \mathrm{K}$ 
Table 1. The grain size of $\mathrm{Pd}_{81} \mathrm{Zr}_{19}$ at different real times and $T=873^{\circ} \mathrm{K}$ (Liu et al 2006).

\begin{tabular}{lrrrrrr}
\hline Grain size (nm) & 4 & 11 & 13 & 15 & $15 \cdot 1$ & $16 \cdot 2$ \\
\hline Real time (s) & 0 & 8333 & 16,666 & 41,666 & 63,333 & 83,333 \\
\hline
\end{tabular}

Table 2. The grain size of RuAl at different real times and $T=1173^{\circ} \mathrm{K}$ (Liu and Mucklich 2001; Liu et al 2006).

\begin{tabular}{lcrrrrr}
\hline Grain size (nm) & $4 \cdot 6$ & 23 & $29 \cdot 2$ & $32 \cdot 3$ & $35 \cdot 3$ & $36 \cdot 9$ \\
\hline Real time (s) & 0 & 1842 & 3421 & 7105 & 10,789 & 17,894 \\
\hline
\end{tabular}

at $1173^{\circ} \mathrm{K}$, are modeled. In figures 1 and 2 the simulated grain sizes are plotted versus the time unit of simulation, i.e. MCS, for both the mentioned materials. As can be seen, the grain sizes of the materials are increased with increasing time unit of simulation. In tables 1 and 2, the grain sizes of the materials at different real times are presented (Liu and Mucklich 2001; Liu et al 2006). Regarding the results of simulation in figures 1 and 2 , and the experimental data in tables 1 and 2, the real time of each grain size can be plotted versus its MCS for each material. These plots are shown in figures 3 and 4 . From the presented data in these figures and using curve-fitting method, the following relationships can be expressed between the real time and MCS for $\mathrm{Pd}_{81} \mathrm{Zr}_{19}$ and $\mathrm{RuAl}$, respectively:

$$
\begin{aligned}
& t_{\mathrm{r}}=2 \times 10^{-9}(M C S-1000)^{3 \cdot 14}, \\
& t_{\mathrm{r}}=10^{-5}(\mathrm{MCS}-1000)^{1.83},
\end{aligned}
$$

where $t_{\mathrm{r}}$ is the real time in seconds.

As can be observed in this study, the relationships are found in power law form. It should be noted that as reported in prior works, for modeling the grain growth of the conventional material the relationship between the real time and MCS has been achieved linearly (Saito and Enomoto 1992; Gao and Thompson 1996). This can be attributed to the higher kinetics of grain growth due to the larger grain boundary area of nano-crystalline materials in comparison with that of the conventional materials (Michels et al 1999; Liu et al 2006). In physical concept, since the nano-crystalline materials have large grain boundary area, its internal energy due to the grain boundary energy is high which leads to more rapid grain growth and higher grain growth kinetics than that of conventional materials. Consequently, to find the higher kinetics, the mathematical power law relationships appear which introduce the higher velocity of grain boundary migration in nano-crystalline materials.

\section{Conclusions}

The grain growth of two nano-crystalline materials, $\mathrm{Pd}_{81} \mathrm{Zr}_{19}$ at $873^{\circ} \mathrm{K}$ and $\mathrm{RuAl}$ at $1173^{\circ} \mathrm{K}$, are modeled using Monte Carlo simulation. The results show that for modeling the nano-scale grain growth the relationship between the real time and time unit of simulation is achieved in power law form. However, in other researches for modeling the grain growth of the conventional materials the relationship has been achieved linearly.

\section{Acknowledgement}

The author would like to thank the Research Board of Sharif University of Technology, for the financial support.

\section{References}

Anderson M P, Srolovitz D J, Grest G S and Sahni P S 1984 Acta Metall. 32783

Christian J W 1965 The theory of transformations in metals and alloys (Oxford: Pergamon Press)

Gao J and Thompson R G 1996 Acta Mater. 444565

Humphreys F J and Hatherly M 1995 Recrystallization and related annealing phenomena (Oxford: Elsevier Science)

Liu K W and Mucklich F 2001 Acta Mater. 49395

Liu F and Kirchheim R 2004 J. Cryst. Growth 264385

Liu F, Yang G, Wang H, Chen Z and Zhou Y 2006 Thermochem. Acta 443212

Michels A, Krill C E, Ehrhardt H, Brringer R and Wu D T 1999 Acta Mater. 472143

Morhac M and Morhacova E 2000 Cryst. Res. Technol. 35117

Saito Y and Enomoto M 1992 ISIJ Int. 32267 